\title{
GATA3 haploinsufficiency causes a rapid deterioration of distortion product otoacoustic emissions (DPOAEs) in mice
}

\author{
M.A.J. van Looij, ${ }^{\mathrm{a}, \mathrm{b}, *}$ H. van der Burg, ${ }^{\mathrm{b}}$ R.S. van der Giessen, ${ }^{\mathrm{b}}$ M.M. de Ruiter, \\ J. van der Wees, ${ }^{\mathrm{c}}$ J.H. van Doorninck, ${ }^{\mathrm{b}}$ C.I. De Zeeuw, ${ }^{\mathrm{b}}$ and G.A. van Zanten ${ }^{\mathrm{a}, \mathrm{d}}$ \\ ${ }^{a}$ Department of ENT, Erasmus Medical Center, PO Box 2060, 3000 CB Rotterdam, The Netherlands \\ ${ }^{\mathrm{b}}$ Department of Neuroscience, Erasmus Medical Center, PO Box 1738, 3000 DR Rotterdam, The Netherlands \\ ${ }^{\mathrm{c}}$ Department of Cell Biology and Genetics, Erasmus MC, PO Box 1738, 3000 DR Rotterdam, The Netherlands \\ ${ }^{\mathrm{d}}$ Department of ENT/Audiology, F.02.504, University Medical Center Utrecht, PO Box 85500, 3508 GA Utrecht, The Netherlands
}

Received 6 February 2005; revised 20 May 2005; accepted 25 May 2005

Available online 1 July 2005

\begin{abstract}
Human HDR (hypoparathyroidism, deafness and renal dysplasia)syndrome is caused by haploinsufficiency of zinc-finger transcription factor GATA3. The hearing loss due to GATA3 haploinsufficiency has been shown to be peripheral in origin, but it is unclear to what extent potential aberrations in the outer hair cells $(\mathrm{OHCs})$ contribute to this disorder. To further elucidate the pathophysiological mechanism underlying the hearing defect in HDR-syndrome, we investigated the OHCs in heterozygous Gata3-knockout mice at both the functional and morphological level. While the signal-to-noise ratios of distortion product otoacoustic emissions (DPOAE) in wild type mice did not change significantly during the first half-year of live, those in the heterozygous Gata3 mice decreased dramatically. In addition, both light microscopic and transmission electron microscopic analyses showed that the number of OHCs containing vacuoles was increased in the mutants. Together, these findings indicate that outer hair cell malfunctioning plays a major role in the hearing loss in HDR-syndrome.
\end{abstract}

(C) 2005 Elsevier Inc. All rights reserved.

Keywords: DPOAE; OHC; Vacuole; HDR-syndrome; EM; Hearing loss; GATA3

Abbreviations: ABR, auditory brainstem response; DPOAE, distortion product otoacoustic emission; EM, electron microscopy; HDR-syndrome, hypoparathyroidism, deafness and renal dysplasia-syndrome; Ht, heterozygous; IHC, inner hair cell; OAE, otoacoustic emission; OHC, outer hair cell; PC, pillar cell; NF, nerve fiber; Wt, wild type.

* Corresponding author. Department of Otorhinolaryngology, Erasmus MC, PO Box 2060, 3000 CB Rotterdam, The Netherlands. Fax: +31 10 4635660 .

E-mail address: m.vanlooij@erasmusmc.nl (M.A.J. van Looij).

Available online on ScienceDirect (www.sciencedirect.com).

\section{Introduction}

In 1992, HDR-syndrome was first described as a new clinical entity, comprising hypoparathyroidism, deafness and renal dysplasia (Bilous et al., 1992).

The sensorineural hearing loss in HDR patients can be both symmetric or asymmetric, and as tested by auditory brainstem response $(\mathrm{ABR})$, conditioned orientation reflex tests or pure tone audiometry, it ranges in level from $40 \mathrm{~dB}$ to $105 \mathrm{~dB}$ (Bilous et al., 1992; Fujimoto et al., 1999; Hasegawa et al., 1997; Lichtner et al., 2000; Muroya et al., 2001). Although it is clear that hearing loss in HDR is usually somewhat more severe at the higher end of the frequency spectrum (Lichtner et al., 2000; Muroya et al., 2001), no systematic audiometric evaluation has yet been made, and the exact pathophysiological mechanism causing the hearing defect remains largely unknown. Genetically, HDR-syndrome is caused by haploinsufficiency of zinc finger transcription factor GATA3 (Van Esch and Bilous, 2001; Van Esch and Devriendt, 2001; Van Esch et al., 2000), which in mice is essential for development of several tissues and organs including the lymphatic system, the sympathetic nervous system, brain, kidney, jaw and inner ear (Lim et al., 2000; Pandolfi et al.; 1995, Pata et al., 1999; Ting et al., 1996; van Doorninck et al., 1999; Zheng and Flavell, 1997). During development, human and murine GATA3 is prominently expressed in virtually all cell types of the inner ear including inner hair cells (IHCs), outer hair cells (OHCs) and supporting cells as well as in various cell types of the central auditory system including neurons in the superior olive and inferior colliculus (Debacker et al., 1999; Karis et al., 2001; Lawoko-Kerali et al., 2002; Rivolta and Holley, 1998; van der Wees et al., 2004; van Doorninck et al., 1999).

In line with HDR-syndrome in humans, heterozygous Gata3 mice show an elevation in their Auditory Brainstem Responsethresholds. Thresholds are elevated by approximately $30 \mathrm{~dB}$, while no interpeak latency differences can be found. In addition, Gata3 
heterozygous mice showed extensive apical cochlear cell loss from young ages onwards, without any morphological abnormalities in the central nervous system. This led us to conclude that hearing loss due to GATA3 haploinsufficiency is peripheral in origin (van der Wees et al., 2004). Building upon the above-mentioned findings, and considering the fact that at light microscopical level outer hair cells (OHCs) degenerated first before subsequent loss of inner hair cells (IHC), pillar cells (PC) and nerve fibers (NF), we hypothesized that $\mathrm{OHC}$ malfunctioning could be an important factor causing hearing loss in GATA3 haploinsufficiency.

The most accurate measure for testing $\mathrm{OHC}$ function is otoacoustic emissions (OAEs) (Schrott et al., 1991). Otoacoustic emissions have been used in mice auditory research for a variety of purposes, among others to study age related hearing loss - AHL (Jimenez et al., 1999; Parham, 1997; Parham et al., 1999), noise induced hearing loss - NIHL - (Jimenez et al., 2001; Vazquez et al., 2001) and effects of hypothyroidism on OHCs (Li et al., 1999). Moreover, OAEs have also been used for auditory phenotyping and to study effects of genetic mutations on the auditory system (Huang et al., 1995, 1998; Konrad-Martin et al., 2001).

To shed light on the potential role of dysfunction of OHCs in the hearing loss found in HDR patients, we investigated the function of these cells in heterozygous Gata3 mice. We investigated functioning of their OHCs by measuring the distortion product otoacoustic emissions (DPOAE) and by further analyzing the morphology of these cells at both the light microscopic and electron microscopic level at young ages (1 to 2 months).

\section{Materials and methods}

\section{Mouse lines/subjects}

Heterozygous Gata3 knockouts (129/C57BL/6 GATA3nlsLacZ mice bred 6 to 8 times into FVB/N background) were compared to wild type mice from the same litter. Animal experiments were performed in accordance with the "Principles of laboratory animal care" (NIH publication No. 86-23, revised 1985) and the guidelines approved by the Erasmus University animal care committee (DEC; protocol No. 138-02-01).

\section{Light microscopy and electron microscopy}

Outer hair cell loss was analyzed at the light microscopic level in 24 cochleae of 7 heterozygous Gata3 and 7 wild type mice, in the age range between 1 and 15 months. The animals were deeply anesthetized with Nembutal and perfused transcardially with $4 \%$ paraformaldehyde in $0.1 \mathrm{M}$ phosphate buffer.

Cochleae were post-fixed in $1 \%$ osmium tetroxide for $1 \mathrm{~h}$, embedded in plastic (Durcupan from Fluka, Buchs, Switzerland), and dissected into 10 'quarter' turns (Bohne and Harding, 1997). In the material of each consecutive 'quarter', both the length of the organ of Corti and the number of present OHCs were determined.

A total of 11 cochleae (left and right ears) obtained from 3 mice of each genotype (of which one was 1 month old and two were 2 months old) were used for analysis of differences between heterozygous and wild type mice at light microscopical level. Cochlear pieces containing those sections of the organ of Corti that are responsive to approximately 4,16 and $32 \mathrm{kHz}$ were used for histological analysis (Ou et al., 2000). Semithin slices (1 $\mu \mathrm{m}$ thick) were cut on a Leica Reicherts Ultratome and counterstained with a
Toluidin blue-staining containing 1\% Toluidin blue, 1\% Methylene blue, $0.2 \%$ Sodiumhydroxide, 1\% Borax, 30\% Ethanediol Ethyleneglycol and $70 \%$ MilliQ water. Slices were examined under a Leica DM-RB microscope. Per cochlear piece, 50 to 163 slices were examined. The number of OHCs containing vacuoles was scored and entered in Excel data files, which were subsequently imported into an SPSS-data file. Statistical analysis of histological data was done using SPSS for Windows version 11.0. An effect was considered significant when $P<0.02$. Non-parametric (Mann-Whitney) tests were used to analyze the difference in numbers of outer hair cells containing vacuoles between genotypes. Apart from this, the effect of age and location of the $\mathrm{OHC}$ in the cochlea on this difference was analyzed.

In order to assess possible additional signs of (outer) hair cell degeneration, such as mitochondrial changes, changes in endoplasmatic reticulum and disappearance of ribosomes ultrathin slices were cut for electron microscopy, and contrasted with uranyl acetate and lead citrate (Oei et al., 2003) of inner ears from three 2month-old mice for each genotype. Slices were analyzed using a Philips CM100 electron microscope.

\section{DPOAE-test procedure}

Per age group, genotype and gender at least 5 mice were subjected to DPOAE measurements (i.e. at least 20 mice were examined per age group). Measurements were performed both longitudinally and transversally (32 mice were tested once, 25 mice twice, 9 mice trice and 7 mice four times). Animals were anesthetized with a mixture of $5 \%$ Fluothane, $\mathrm{N}_{2} \mathrm{O}$ and $\mathrm{O}_{2}$. After induction of anesthesia, mice were placed on a heating pad (Thermocomfort, Brisk BV, Venray, The Netherlands), to maintain constant body temperature. In each mouse, the right and left ears were consecutively tested. Cerumen obstruction and infection were excluded prior to experiments by otoscopically inspecting ear canals and eardrums. Mice suffering from obvious outer or middle ear problems were temporarily excluded from experiments. To equalize the anesthesia induced increase in middle ear pressure, the pars flaccida of the tympanum was perforated using a Microlance ${ }^{\circledR}$ needle. The success rate of this procedure was high. Occasionally, however, the perforation was unsuccessful or closed during measurements, resulting in a decrease of signal-to-noise ratios of emissions. If so, a second tympanocentesis was performed. Repeated measurements (and ear drum perforations) in individual mice did not influence DPOAE levels; an illustrative example of this is given in Fig. 1. Signal-to-noise ratios of two 5-month-old mice are compared, one after a single, and the other after four test sessions. Next, foam stripped Etymotic ${ }^{\circledR}$ ER10C-14C ear tips were fitted in the external ear canals to enable recording of DPOAE.

\section{DPOAE measurements}

2f1-f2 DPOAEs were measured using a commercially available recording system (DP 2000 - Starkey). After introduction of the probe and prior to actual recordings, calibration of the stimulation conditions was done over a $0.1-20 \mathrm{kHz}$ frequency range. The primary frequency ratio (f2/f1) was 1.2 (Le Calvez et al., 1998b). The targeted primary tone levels, L1/L2, were $65 / 55$ dB SPL. Data were collected with f2 at 6.8, 8.0, 9.5, 11.3, 13.5 and $16.0 \mathrm{kHz}$. All experiments were performed in a quiet but not sound treated room. Recordings were repeated (at least 6 times). 


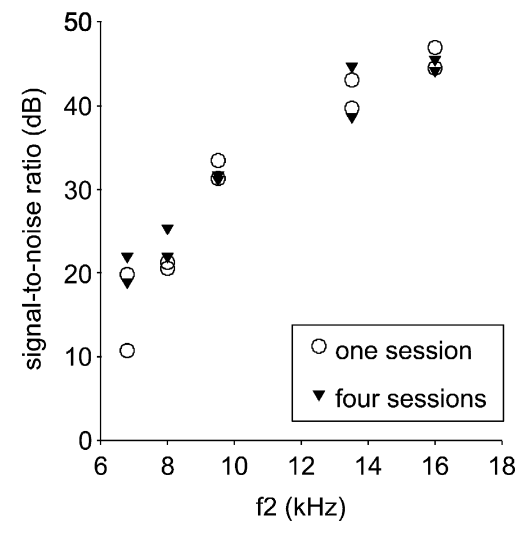

Fig. 1. DPOAE signal-to-noise ratios (dB) of two 5-month-old wild type mice; open circles indicate results after a single measurement, closed triangles represent results after four test sessions. Data for both ears are represented. Repeated measurements and paracentesis in individual mice did not influence DPOAE levels.

\section{DPOAE data processing and analyses}

DPOAE data processing was done using SPSS for Windows version 10.0. Raw data, as stored in the database of the DPOAE recording system, were imported into SPSS-data files. Data were included for statistical analysis after a strict selection as to quality of calibration curves of both stimulus channels, on noise floor and stimulus condition quality during recordings. Symmetry criteria of the calibration curves of both loudspeakers in the probe were used for selection of good quality recordings. Furthermore, recordings with calibration curves showing a probable air leakage or occlusion of probe channels by the ear canal wall were excluded, as were recordings with background noise levels over $-2 \mathrm{~dB}$ SPL and recordings with stimulation levels differing too much from targeted levels (more than $15 \mathrm{~dB}$ ). Finally, only recordings with primary tone level differences ranging from -1 to $12 \mathrm{~dB}$ were included. Table 1 displays the number of mice per age group, genotype and frequency (f2) after strict selection of data. For the f $2=11.3 \mathrm{kHz}$, target levels of the primaries often failed to meet the selection criteria. Therefore, this frequency was excluded from further statistical analysis. Subsequently, of every individual mouse, the emission with the best signal-to-noise ratio per age, per frequency, and per ear was selected for analysis. Since each individual mouse was measured on both ears, we analyzed ear effects prior to further analyses. Within individual mice, a significant correlation existed between right and left ears (Pearson's correlation coefficient $=0.81$, significance $<0.0005)$. Therefore, data were aggregated for each mouse (means of right and left ears), at each age and frequency, for further analysis of genotype and gender influence. Statistical analysis of DPOAE data was done using SPSS for Windows version 10.0. An effect was considered significant when $P<0.02$.

Effects of genotype on signal-to-noise ratio's were analyzed, as were age effects, and gender effects.

\section{Results}

\section{Light microscopy}

Quantitative analyses of the OHCs revealed a progressive loss of apical outer hair cells in heterozygous mice from 1 month onward, significantly preceding and exceeding loss in wild types. At the same time, some degeneration occurred at the basal turn of the cochlea, but the level of this process was comparable to that in wild type mice. By the age of 9 months, in mutants, nearly all OHCs had disappeared, while in the wild types, OHCs were still only affected at the base of the cochlea (Figs. 2A and B). Thus, Gata3 haploinsufficiency induced degeneration started at the apex and ultimately progressed into the middle turns of the cochlea.

To investigate effect of Gata3 haploinsufficiency on the morphological structure of outer hair cells from heterozygous Gata3 mice, we analyzed semithin plastic sections and compared them to sections from wild types. We examined 1151 slices, representing 11 cochleae of 6 mice, at light microscopic level. The estimated width of $\mathrm{OHCs}$ in the cochlear regions examined in our study was approximately $5 \mu \mathrm{m}$, so that a maximum of 5 semithin slices per OHC may have been included in histological analysis. No compelling differences between heterozygous mice and wild type controls were found other than numbers of OHCs showing vacuoles. OHCs in heterozygous mice contained vacuoles more often than those in controls, an illustrative example of this can be seen in Figs. 3A and B. In heterozygous mice, vacuoles were found throughout the cell, whereas when present in wild type mice, they were predominantly seen in the apical region.

The difference in numbers of OHCs containing vacuoles was age-dependent: at 1 month of age, this number significantly differed between genotypes for the first row of OHCs only (Mann-Whitney, $P<0.02$ ), whereas at 2 months of age, the difference was significant (Mann-Whitney, $P<0.02$ ) for all three rows (see Fig. 4A).

Analysis within genotypes demonstrated an increase in the number of cells with vacuoles in the second as compared to the first month in heterozygous mice. This increase was significant for all three rows of OHCs at $P<0.02$ (see Fig. 4A). In wild types, numbers of cells containing vacuoles were low constantly over the observed age range.

The significant age-dependent increase in affected OHCs in heterozygous mice is mainly due to the highly significant increase in vacuoles in all rows of OHCs in the apical pieces (MannWhitney, $P<0.001)$. In the mid-cochlear pieces, a similar trend was seen, but no significant differences could be demonstrated (see Fig. 4B).

In conjunction, our data show that GATA3 haploinsufficiency induces $\mathrm{OHC}$ degeneration, especially in the apical regions of the

Table 1

Number of mice per age group, genotype and frequency (f2) after selection of data on DPOAE recording-quality criteria

\begin{tabular}{|c|c|c|c|c|c|c|c|c|c|c|c|c|}
\hline \multirow{3}{*}{$\begin{array}{l}\text { Age } \\
\text { (months) }\end{array}$} & \multicolumn{12}{|c|}{$\mathrm{f} 2$ frequencies $(\mathrm{kHz})$ and genotypes } \\
\hline & \multicolumn{2}{|c|}{6.8} & \multicolumn{2}{|l|}{8.0} & \multicolumn{2}{|l|}{9.5} & \multicolumn{2}{|c|}{11.3} & \multicolumn{2}{|c|}{13.5} & \multicolumn{2}{|c|}{16.0} \\
\hline & wt & ht & wt & ht & wt & ht & wt & ht & wt & ht & wt & ht \\
\hline 1.0 & 8 & 9 & 9 & 11 & 9 & 11 & & 1 & 6 & 9 & 9 & 11 \\
\hline 2.0 & 7 & 10 & 7 & 11 & 7 & 11 & 2 & 2 & 6 & 10 & 6 & 10 \\
\hline 3.0 & 9 & 12 & 10 & 12 & 10 & 12 & 3 & 2 & 9 & 12 & 10 & 12 \\
\hline 4.0 & 9 & 8 & 10 & 8 & 10 & 8 & 5 & 3 & 6 & 8 & 9 & 8 \\
\hline 5.0 & 11 & 10 & 11 & 11 & 11 & 11 & & 5 & 11 & 10 & 11 & 11 \\
\hline 6.0 & 11 & 10 & 11 & 10 & 11 & 10 & 3 & 4 & 11 & 10 & 11 & 10 \\
\hline 7.0 & 5 & 8 & 7 & 9 & 7 & 9 & & 3 & 5 & 9 & 7 & 9 \\
\hline
\end{tabular}

For the $\mathrm{f} 2=11.3 \mathrm{kHz}$, only a limited number of data met the selection criteria, therefore, this frequency was excluded from further statistical analysis (wt; wild type, ht; heterozygous). 


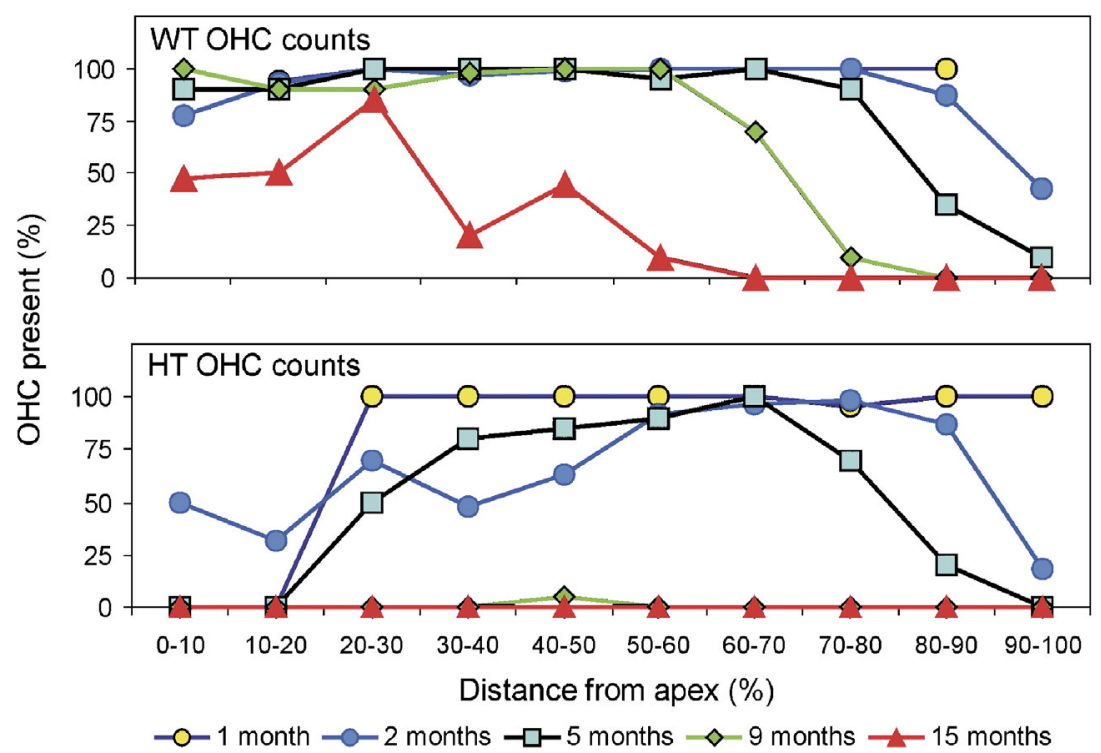

Fig. 2. Presence of outer hair cells (OHC) in cochleae of wild type (upper panel) and heterozygous (lower panel) Gata3 mice of 1,2,5,9 and 15 months of age. Markers indicate the percentage of cells present in the 10 different cochlear pieces, plotted against the distance to the apex of the analyzed cochlear part. Hair cells responsive to $4 \mathrm{kHz}, 8 \mathrm{kHz}, 16 \mathrm{kHz}$ and $32 \mathrm{kHz}$ tones are found at approximately $25 \%, 40 \%, 60 \%$ and $75 \%$ distance from the apex, respectively (Ou et al., 2000). OHCs in both the apical and basal cochlear regions degenerate in heterozygous mice, and progressively so with age. Wild type controls in contrast, are only affected by (normal) loss of basal outer hair cells (previously, data were presented partially in van der Wees et al., 2004).
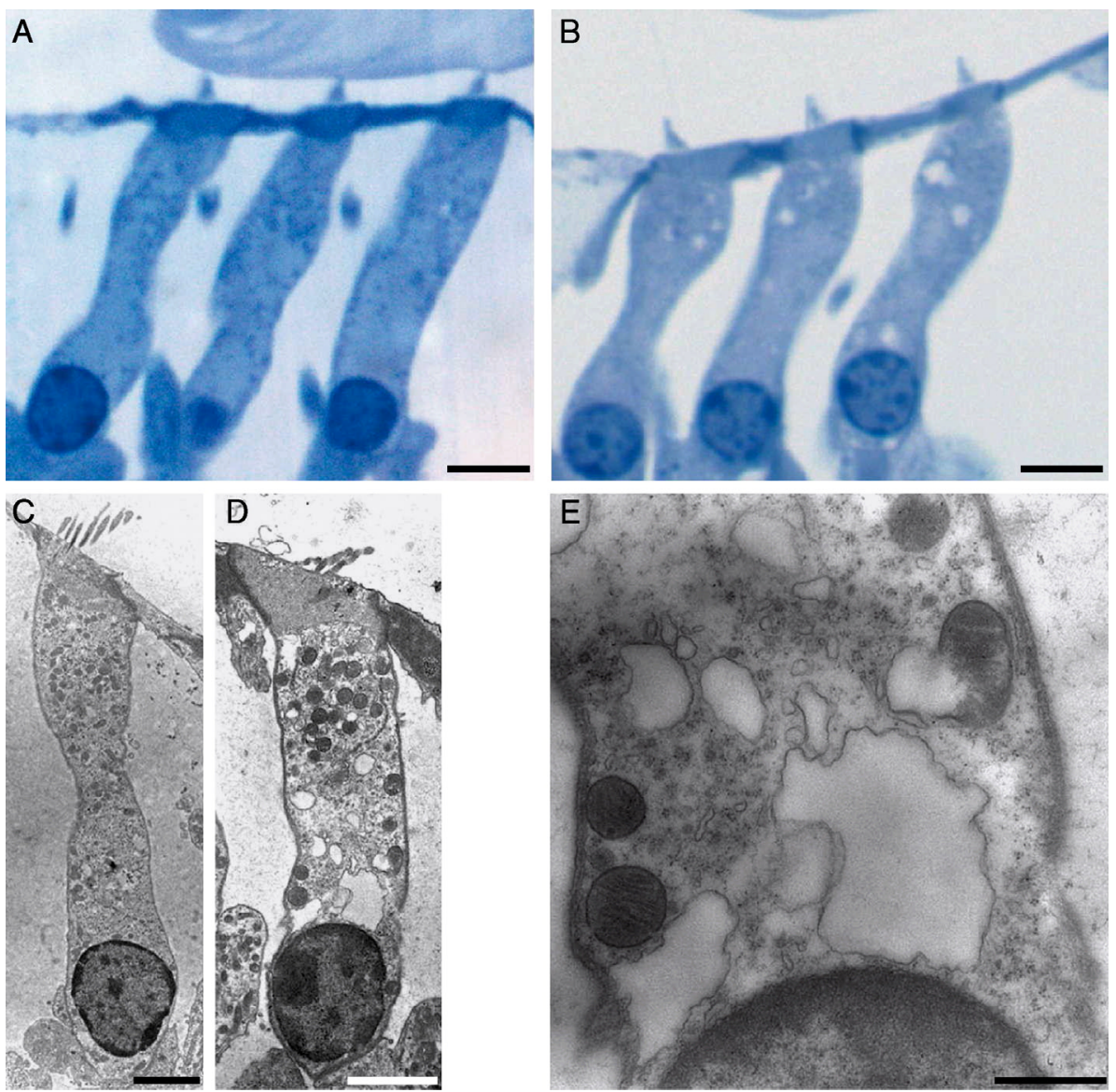

Fig. 3. Vacuolization in Gata3 heterozygous OHCs. Plastic sections of normal OHCs from a 2-month-old wild type mouse (A) and of abnormal OHCs, with vacuoles in all three $\mathrm{OHC}$ rows, from a 2-month-old heterozygous Gata3 mouse (B). Electronmicroscopy of a normal wild type (C) and a Gata3 +/- OHC (D) shows multiple vacuoles in the mutant $\mathrm{OHC}$ that otherwise looks normal. Panel $\mathrm{E}$ is a magnification of panel D and shows the irregular shape of the unfilled vacuoles that have a single layer membrane. Scale bars are $2 \mu \mathrm{m}$ for panels A-D and $0.5 \mu \mathrm{m}$ for panel $\mathrm{E}$. 
A

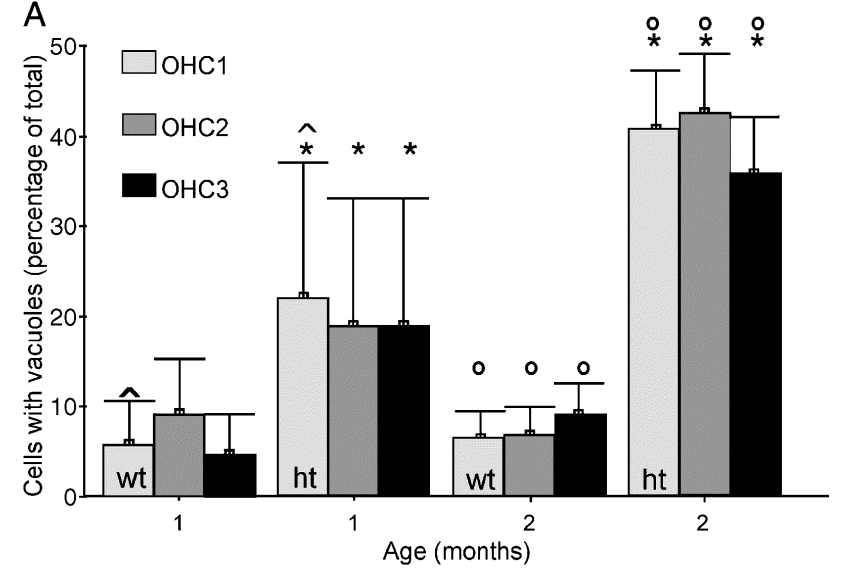

B

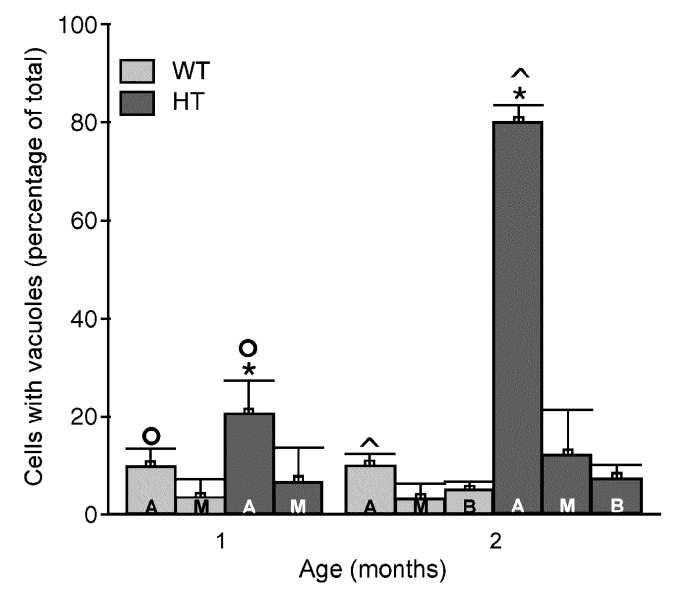

Fig. 4. (A) Percentages of cells containing vacuoles versus age and genotype (wt; wild type, ht; heterozygous). Numbers of OHCs containing vacuoles significantly differed between genotypes for the first row of OHCs at 1 month of age $\left(^{\wedge}\right)$, at 2 months of age, this difference was significant for all three rows (o). Analysis within genotypes demonstrated an increase in the number of cells with vacuoles in the second as compared to the first month in heterozygous mice. This increase was significant for all three rows of OHCs at $P<0.02(*)$. In wild types, less than $10 \%$ of cells contained vacuoles. Error bars indicate 95\% confidence intervals. (B) Percentages of cells containing vacuoles versus age per genotype and cochlear localization. Analysis of influence of cochlear localization demonstrated the increase in the number of cells with vacuoles as described in heterozygous mice in Fig. $2 \mathrm{~A}$, to be due to the highly significant increase in vacuoles in all rows of OHCs in the apical pieces in the second as compared to the first month of age $\left(^{*}\right)$ (Mann-Whitney, $P<0.001$ ). No significant differences could be demonstrated in the mid-cochlear or basal pieces. Numbers of cells containing vacuoles in the cochlear apex, however, differed significantly between genotypes, both at 1 (o) and 2 months $\left(^{\wedge}\right)$ of age (Mann-Whitney, $P<0.02$ ). Error bars indicate $95 \%$ confidence intervals. $\mathrm{A}=$ apical, $\mathrm{M}=$ mid-cochlear, $\mathrm{B}=$ basal.

cochlea, in mice as young as 1 month of age. These deleterious effects increase with increasing age.

\section{Electron microscopy}

To closer examine the nature of the observed vacuoles in the $\mathrm{OHCs}$, we investigated them at the ultrastructural level. The vacuoles were irregularly shaped and surrounded by a single layer membrane (Figs. 3D and E). The OHCs did not show any sign of apoptosis or immediate cell death such as pyknotic nuclei or abnormal mitochondria. Thus, the first sign of GATA3-driven degeneration of OHCs seems to be the appearance of irregularly shaped vacuoles, which can be observed at both the light microscopic level and at electron microscopic level.

\section{Otoacoustic emissions}

Outer hair cell functionality was examined by measuring DPOAE levels in both Gata3 heterozygous and wild type mice at various ages. DPOAE signal-to-noise ratios differed significantly between Gata3 heterozygous mice and wild types when data were pooled over all f2's and ages (Mann-Whitney Test, $P<$ 0.001 ) (Fig. 5). When separate ages and f2's were analyzed, this significance was consistent (at the $P<0.02$ level) for groups larger than 6 (see Table 1), with the sole exception of 7-month-old mice at $9.5 \mathrm{kHz}(P=0.023)$. Emission levels in the $\mathrm{f} 2$ frequency range from $6.8 \mathrm{kHz}$ to $16.0 \mathrm{kHz}$ remained fairly constant in wild type mice during the first 7 months of life, whereas in the mutants, signal-to-noise ratios rapidly decreased during this period (Figs. 5 and 6). At 7 months of age, signal-to-noise ratios of DPOAEs in heterozygous mice no longer differed from $0 \mathrm{~dB}$. Signal-to-noise ratios did not differ significantly between genders for pooled data ( $t$ test, $P>0.2$ ), or within specific age groups ( $t$ tests, $P>0.02$ ).

In sum, our acousto-physiological analyses demonstrated a rapid deterioration of $\mathrm{OHC}$ functionality in Gata3 heterozygous mice, starting apically in the cochlea and progressing onto its base.

\section{Discussion}

Here, we show that young heterozygous Gata3 mice generate abnormal Distortion Product Oto-Acoustic Emissions (DPOAEs) and that their outer hair cells (OHCs) are commonly affected by vacuoles. Together, these data raise the possibility that dysfunctional OHCs form one of the prime causes of hearing loss in these mutants. Since HDR patients, who suffer from the same haploinsufficiency as these mouse mutants, suffer from hearing loss as well, this pathological mechanism may also hold for HDR patients.

Outer hair cells are affected by both histological and physiological aberrations

Otoacoustic emissions (OAEs) are the best means for testing OHC function in vivo (Schrott et al., 1991). In our present study, distortion product otoacoustic emissions were recorded in 1-7month-old Gata3 heterozygous mice and in age- and sex-matched controls. In both genotypes, the loudest emissions were found in the higher end of the frequency range $(6.8 \mathrm{kHz}-16 \mathrm{kHz})$. Emission levels decreased rapidly over time in heterozygous knockout mice, whereas in wild type controls, they remained fairly constant during the first 7 months of life. Signal-to-noise emission levels in the $\mathrm{FVB} / \mathrm{N}$ controls in this study are equal to, or even slightly better than emission levels in the CBA/J mouse model, which is considered a well hearing strain until relatively late in life (Parham et al., 1999). During DPOAE recordings, we used gas anesthesia, since this type of narcosis provides flexibility (e.g. short-term anesthesia), despite the known increase in middle ear pressure caused by $\mathrm{N}_{2} \mathrm{O}$, necessitating paracentesis. Moreover, we demonstrated that repeated measurements in itself did not influence DPOAE levels (Fig. 1). Thus, in contrast to the commonly used 

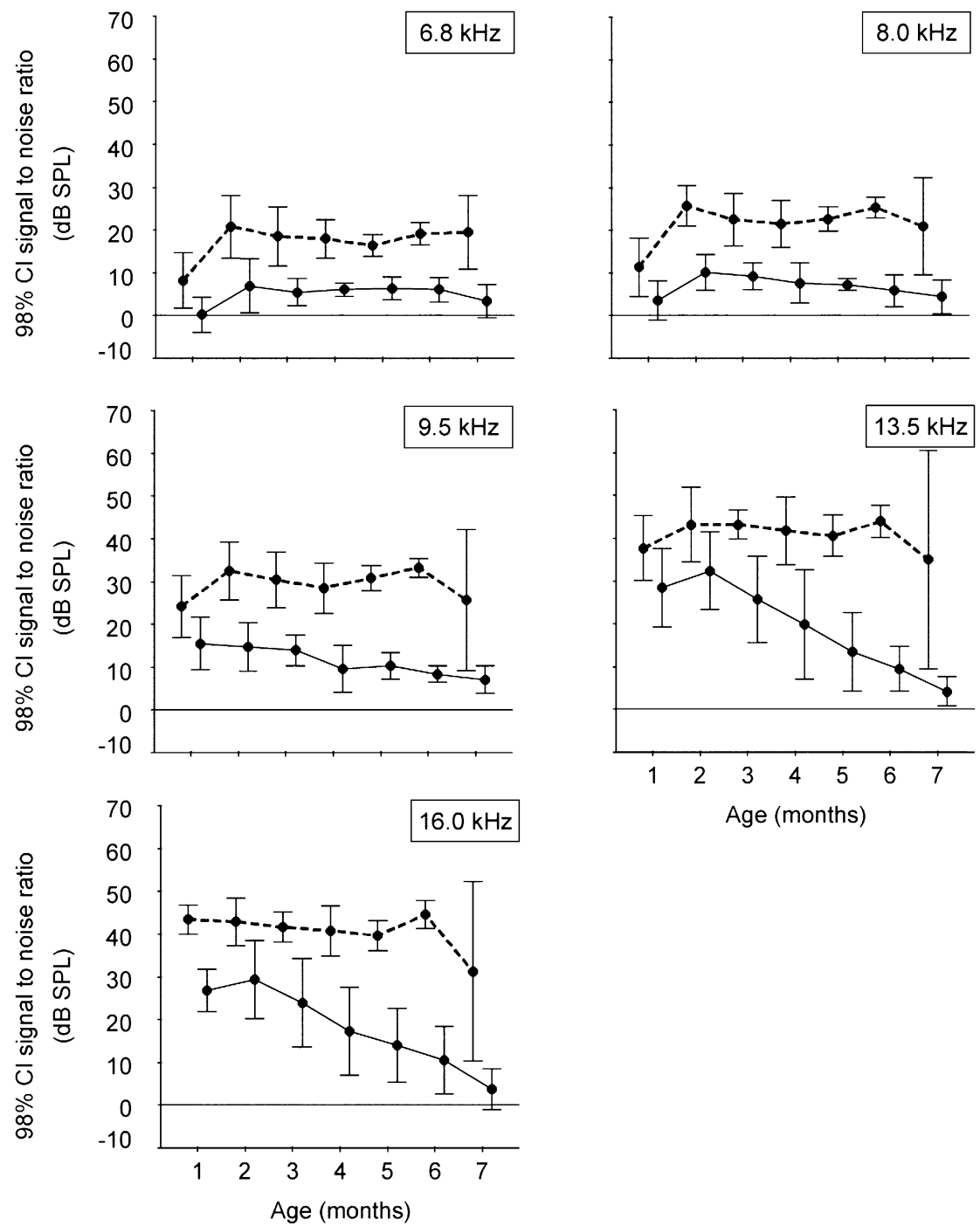

Fig. 5. 98\% confidence intervals of DPOAE signal-to-noise ratios (dB SPL) at f2's 6.8-16.0 kHz for wild type (discontinuous line) and heterozygous (continuous line) mice aged 1 to 7 months. At all ages and frequencies, emissions in wild types were stronger than in heterozygous mice. At 7 months of age, hardly any emissions could be generated in heterozygous knockouts, whereas signal-to-noise ratios in wild types remained fairly constant. Signal-to-noise ratios at $11.3 \mathrm{kHz}$ are not represented graphically, since statistical analysis was unreliable due to small numbers of data (see Table 1).
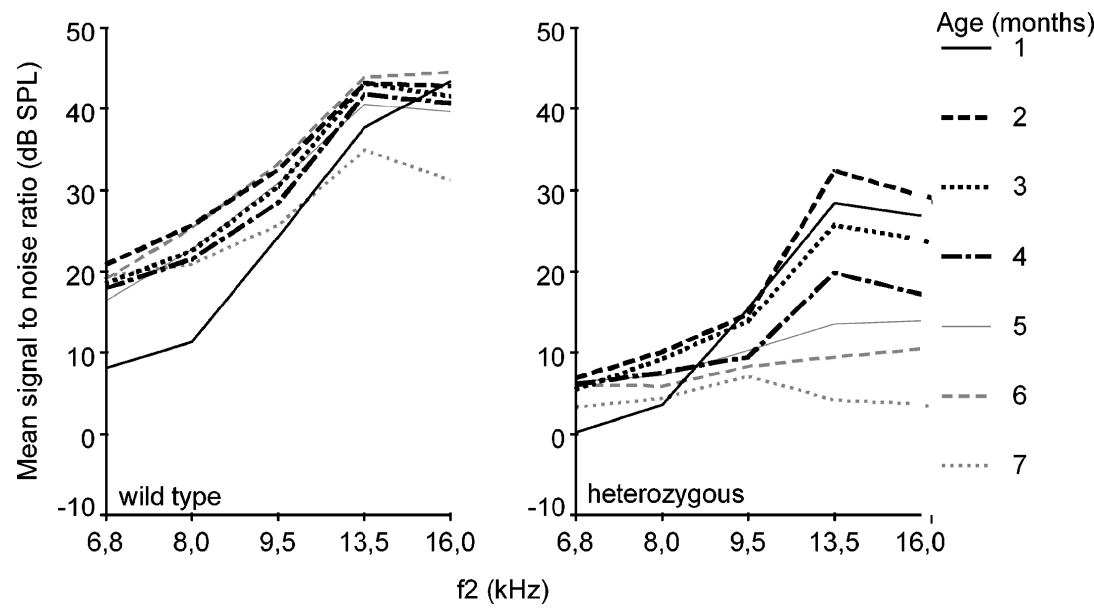

Fig. 6. Signal-to-noise ratios (dB SPL) of 1-7-month-old wild type and heterozygous mice in the f2 frequency range 6.8 to $16.0 \mathrm{kHz}$. During the first $7 \mathrm{months}$ of life, emission levels remain fairly constant in wild type mice, whereas in heterozygous mice, signal-to-noise ratios rapidly decrease. 
intraperitoneal or intramuscularly anesthesia (e.g. Sun and Kim, 1999; Jimenez et al., 2001; Konrad-Martin et al., 2001), gas anesthesia may be quite feasible for (longitudinal) DPOAE recordings.

Heterozygous mice also differed from controls in numbers of $\mathrm{OHCs}$ containing vacuoles, increasingly so with age. Curiously, no gross abnormalities were found in other cochlear cell types expressing GATA3. Further histological analysis of $\mathrm{OHCs}$ by electron microscopy excluded other changes at the cellular level such as apoptosis. Vacuoles are frequently seen as the first signs of (hair) cell death (Vetter et al., 1996). At 2 months of age, the numbers of outer hair cells containing vacuoles in heterozygous mice were especially high in the apical region of the cochleae, which is responsive to frequencies of approximately $4 \mathrm{kHz}(\mathrm{Ou}$ et al., 2000), whereas in the middle (responsive to $16 \mathrm{kHz}$ ) and basal regions (responsive to $32 \mathrm{kHz}$ ), these numbers remained low. These findings are in agreement with the outcomes of the DPOAE measurements, which showed that the loudest emissions were found in the vacuole-free frequency regions. Thus, although the current data do not allow us to conclude that there is a direct causal relation between the deficits in DPOAEs and the occurrence of vacuoles in the OHCs, both findings suggest that functional deficits in OHCs may contribute to hearing loss in HDR-syndrome.

\section{Role of outer hair cells in hearing loss in HDR}

Our findings that GATA3 haploinsufficiency induces an agedependent loss of $\mathrm{OHC}$ function as well as concomitant histological anomalies in OHCs raise the question as to whether the OHCs can be held solely responsible for the hearing loss in HDR-syndrome. Although at present it is not possible to answer this question conclusively, several findings suggest that we cannot exclude this possibility either.

First, deficits in both DPOAEs and ABRs start to occur in the first few months of life (see also van der Wees et al., 2004).

Second, DPOAE signal-to-noise ratios in heterozygous Gata3 mice as compared to controls are lower for all $\mathrm{f} 2$ frequencies in the $6.8-16.0 \mathrm{kHz}$ range, agreeing with the fact that ABR-thresholds in these mice were elevated for all tested frequencies $(4-32 \mathrm{kHz})$ in the age range of 1-19 months as compared to controls. Moreover, ABR-thresholds are worse at $4 \mathrm{kHz}$, compared to $16 \mathrm{kHz}$ for ages up to 19 months, this corresponds to lower DPOAE signal-to-noise ratio's in the lower end of the frequency range described here.

Third, ABR-thresholds in heterozygous mice are on average elevated by approximately $30 \mathrm{~dB}$ (van der Wees et al., 2004), which agrees well with numerous other animal models in which there is a presumptive selective deficit of the OHCs. For example, Kanamycin sulfate treatment, causing complete loss of OHCs, with preservation of IHCs, results in threshold shifts of $40 \mathrm{~dB}$ and more in chinchillas (Woolf et al., 1981). In the same species, the first approximate $30 \mathrm{~dB}$ of noise-exposure induced permanent threshold shifts is induced by primarily outer hair cell losses (Hamernik et al., 1989). On the other hand, neomycin in combination with acoustic trauma causes decreases in cochlear sensitivity amounting up to $62 \mathrm{~dB}$, averaged across all frequencies, in guinea pigs with losses of cochlear OHCs close to $100 \%$ (Brown et al., 1978). Unfortunately, the effect of the used treatment on other cochlear cell types was not described. Finally, homozygous Kit $W$-v mice $(\mathrm{Wv} / \mathrm{Wv})$, which have normal IHCs but lack $98 \%$ of cochlear OHCs, have an elevation of ABR-thresholds up to about $50 \mathrm{~dB}$, as compared to control mice (Schrott et al., 1990).
It should be noted that the exact correlation between DPOAEs and ABR-thresholds remains to be established. DPOAEs were below the level of detection in laboratory animals such as mice and chinchilla's when ABR-threshold shifts exceeded approximately 40 dB (e.g. Kakigi et al., 1998; Le Calvez et al., 1998a), however, when tentatively correlating DPOAE levels to ABR-thresholds variances were fairly large and no significant correlation was established so far. When considering our data, we find that the best DPOAE signal-to-noise ratios in heterozygous Gata3 mice were recorded in the upper half of the tested frequency spectrum during the first months, when ABR-thresholds of corresponding frequencies ( 8 and $16 \mathrm{kHz}$ ) ranged approximately between 30 and $50 \mathrm{~dB}$ SPL. Moreover, in wild type controls, DPOAE signal-to-noise ratios were high and remained fairly constant over the time span presented in this paper, where ABR-thresholds did on average not exceed $40 \mathrm{~dB}$ SPL during the first 7 months of life for the 4-16 $\mathrm{kHz}$ range.

Thus, taken together, we can conclude that it appears likely that OHC dysfunction caused by GATA3 haploinsufficiency contributes to hearing loss in HDR-syndrome, but it remains to be demonstrated whether it is the sole cause.

\section{Conclusions}

GATA3 haploinsufficiency is known to cause hearing loss in both humans affected by the HDR-syndrome and mice. The goal of the present study was to elucidate the role of outer hair cells in this process. We demonstrated that GATA3 haploinsufficiency causes a rapid deterioration of $\mathrm{OHC}$ function in mice. Apical OHCs show early signs of cell degeneration in affected mice as young as 1 to 2 months in that they exhibit multiple vacuoles and produce abnormal emissions. We conclude that outer hair cell malfunctioning can play a major role in the hearing loss in HDR-syndrome.

\section{Acknowledgments}

This work was supported by the Heinsius Houbolt Foundation and the Dutch Organization for Scientific Research (NWO; CDZ) and Neuro-Bsik (CDZ). The authors would like to thank Elize Haasdijk for technical support.

\section{References}

Bilous, R.W., Murty, G., Parkinson, D.B., Thakker, R.V., Coulthard, M.G., Burn, J., Mathias, D., Kendall-Taylor, P., 1992. Brief report: autosomal dominant familial hypoparathyroidism, sensorineural deafness, and renal dysplasia. N. Engl. J. Med. 327, 1069-1074.

Bohne, B.A., Harding, G.W., 1997. Processing and analyzing the mouse temporal bone to identify gross, cellular and subcellular pathology. Hear. Res. 109, 34-45.

Brown, J.J., Brummett, R.E., Meikle, M.B., Vernon, J., 1978. Combined effects of noise and neomycin. Acta Oto-Laryngol. 86, 394-400.

Debacker, C., Catala, M., Labastie, M.C., 1999. Embryonic expression of the human GATA-3 gene. Mech. Dev. 85, 183-187.

Fujimoto, S., Yokochi, K., Morikawa, H., Nakano, M., Shibata, H., Togari, H., Wada, Y., 1999. Recurrent cerebral infarctions and del(10) (p14p15.1) de novo in HDR (hypoparathyroidism, sensorineural deafness, renal dysplasia) syndrome. Am. J. Med. Genet. 86, 427-429.

Hamernik, R.P., Patterson, J.H., Turrentine, G.A., Ahroon, W.A., 1989. The quantitative relation between sensory cell loss and hearing thresholds. Hear. Res. 38 (3), 199-211. 
Hasegawa, T., Hasegawa, Y., Aso, T., Koto, S., Nagai, T., Tsuchiya, Y., Kim, K.C., Ohashi, H., Wakui, K., Fukushima, Y., 1997. HDR syndrome (hypoparathyroidism, sensorineural deafness, renal dysplasia) associated with del(10)(p13). Am. J. Med. Genet. 73, 416-418.

Huang, J.M., Money, M.K., Berlin, C.I., Keats, B.J., 1995. Auditory phenotyping of heterozygous sound-responsive $(+/ \mathrm{dn})$ and deafness (dn/dn) mice. Hear. Res. 88, 61-64.

Huang, J.M., Berlin, C.I., Lin, S.T., Keats, B.J., 1998. Low intensities and 1.3 ratio produce distortion product otoacoustic emissions which are larger in heterozygous $(+/ \mathrm{dn})$ than homozygous $(+/+)$ mice. Hear. Res. $117,24-30$

Jimenez, A.M., Stagner, B.B., Martin, G.K., Lonsbury-Martin, B.L., 1999. Age-related loss of distortion product otoacoustic emissions in four mouse strains. Hear. Res. 138, 91-105.

Jimenez, A.M., Stagner, B.B., Martin, G.K., Lonsbury-Martin, B.L., 2001. Susceptibility of DPOAEs to sound overexposure in inbred mice with AHL. J. Assoc. Res. Otolaryngol. 2, 233-245.

Kakigi, A., Hirakawa, H., Harel, N., Mount, R.J., Harrison, R.V., 1998. Comparison of distortion-product and transient evoked otoacoustic emissions with ABR threshold shift in chinchillas with ototoxic damage. Auris, Nasus, Larynx 25, 223-232.

Karis, A., Pata, I., van Doorninck, J.H., Grosveld, F., de Zeeuw, C.I., de Caprona, D., Fritzsch, B., 2001. Transcription factor GATA-3 alters pathway selection of olivocochlear neurons and affects morphogenesis of the ear. J. Comp. Neurol. 429, 615-630

Konrad-Martin, D., Norton, S.J., Mascher, K.E., Tempel, B.L., 2001 Effects of PMCA2 mutation on DPOAE amplitudes and latencies in deafwaddler mice. Hear. Res. 151, 205-220.

Lawoko-Kerali, G., Rivolta, M.N., Holley, M., 2002. Expression of the transcription factors GATA3 and Pax2 during development of the mammalian inner ear. J. Comp. Neurol. 442, 378-391.

Le Calvez, S., Avan, P., Gilain, L., Romand, R., 1998a. CD1 hearingimpaired mice: I. Distortion product otoacoustic emission levels, cochlear function and morphology. Hear. Res. 120, 37-50.

Le Calvez, S., Guilhaume, A., Romand, R., Aran, J.M., Avan, P., 1998b. CD1 hearing-impaired mice: II. Group latencies and optimal f2/f1 ratios of distortion product otoacoustic emissions, and scanning electron microscopy. Hear. Res. 120, 51-61.

Li, D., Henley, C.M., O’Malley Jr., B.W., 1999. Distortion product otoacoustic emissions and outer hair cell defects in the hyt/hyt mutant mouse. Hear. Res. 138, 65-72.

Lichtner, P., Konig, R., Hasegawa, T., Van Esch, H., Meitinger, T. Schuffenhauer, S., 2000. An HDR (hypoparathyroidism, deafness, renal dysplasia) syndrome locus maps distal to the DiGeorge syndrome region on 10p13/14. J. Med. Genet. 37, 33-37.

Lim, K.C., Lakshmanan, G., Crawford, S.E., Gu, Y., Grosveld, F., Engel, J.D., 2000. Gata3 loss leads to embryonic lethality due to noradrenaline deficiency of the sympathetic nervous system. Nat. Genet. 25, $209-212$.

Muroya, K., Hasegawa, T., Ito, Y., Nagai, T., Isotani, H., Iwata, Y., Yamamoto, K., Fujimoto, S., Seishu, S., Fukushima, Y., Hasegawa, Y., Ogata, T., 2001. GATA3 abnormalities and the phenotypic spectrum of HDR syndrome. J. Med. Genet. 38, 374-380.

Oei, M.L.Y.M. Segenhout, M. Dijk, F. Albers. F.W.J., 2003. Ultrastructural morphology of the guinea pig ear after systemic gentamycin application. "Morphology and electrophysiology of the vestibular organ in the guinea pig," Thesis University of Groningen, pp. 57-70.

Ou, H.C., Harding, G.W., Bohne, B.A., 2000. An anatomically based frequency place map for the mouse cochlea. Hear. Res. 3460, 1-7.

Pandolfi, P.P., Roth, M.E., Karis, A., Leonard, M.W., Dzierzak, E.
Grosveld, F.G., Engel, J.D., Lindenbaum, M.H., 1995. Targeted disruption of the GATA3 gene causes severe abnormalities in the nervous system and in fetal liver haematopoiesis. Nat. Genet. 11, $40-44$.

Parham, K., 1997. Distortion product otoacoustic emissions in the C57BL/6J mouse model of age-related hearing loss. Hear. Res. 112, $216-234$.

Parham, K., Sun, X.M., Kim, D.O., 1999. Distortion product otoacoustic emissions in the $\mathrm{CBA} / \mathrm{J}$ mouse model of presbycusis. Hear. Res. 134, $29-38$

Pata, I., Studer, M., van Doorninck, J.H., Briscoe, J., Kuuse, S., Engel, J.D., Grosveld, F., Karis, A., 1999. The transcription factor GATA3 is a downstream effector of Hoxb1 specification in rhombomere 4. Development $126,5523-5531$.

Rivolta, M.N., Holley, M.C., 1998. GATA3 is downregulated during hair cell differentiation in the mouse cochlea. J. Neurocytol. 27, 637-647.

Schrott, A., Stephan, K., Spoendlin, H., 1990. Auditory brainstem response thresholds in a mouse mutant with selective outer hair cell loss. Eur. Arch. Otolaryngol. 247, 8-11.

Schrott, A., Puel, J.L., Rebillard, G., 1991. Cochlear origin of 2f1-f2 distortion products assessed by using 2 types of mutant mice. Hear. Res. $52,245-253$

Sun, X.M., Kim, D.O., 1999. Adaptation of 2f1-2f2 distortion product otoacoustic emission in young-adult and old CBA and C57 mice. J. Acoust. Soc. Am. 105 (6), 3399-3409.

Ting, C.N., Olson, M.C., Barton, K.P., Leiden, J.M., 1996. Transcription factor GATA-3 is required for development of the T-cell lineage. Nature $384,474-478$.

van der Wees, J., van Looij, M.A.J., de Ruiter, M.M., Elias, H., van der Burg, H., Liem, S.S., Kurek, D., Engel, J.D., Karis, A., van Zanten, G.A., de Zeeuw, C.I., Grosveld, F.G., van Doorninck, J.H., 2004 Hearing loss following GATA3 haploinsufficiency is caused by cochlear disorder. Neurobiol. Dis. 16, 169-178

van Doorninck, J.H., van der Wees, J., Karis, A., Goedknegt, E., Engel, J.D., Coesmans, M., Rutteman, M., Grosveld, F., De Zeeuw, C.I., 1999. GATA-3 is involved in the development of serotonergic neurons in the caudal raphe nuclei. J. Neurosci. 19, RC12.

van Esch, H., Bilous, R.W., 2001. GATA3 and kidney development: why case reports are still important. Nephrol., Dial., Transplant 16, $2130-2132$.

van Esch, H., Devriendt, K., 2001. Transcription factor GATA3 and the human HDR syndrome. Cell. Mol. Life Sci. 58, 1296-1300.

van Esch, H., Groenen, P., Nesbit, M.A., Schuffenhauer, S., Lichtner, P., Vanderlinden, G., Harding, B., Beetz, R., Bilous, R.W., Holdaway, I., Shaw, N.J., Fryns, J.P., Van de Ven, W., Thakker, R.V., Devriendt, K., 2000. GATA3 haplo-insufficiency causes human HDR syndrome. Nature 406, 419-422.

Vazquez, A.E., Luebke, A.E., Martin, G.K., Lonsbury-Martin, B.L., 2001. Temporary and permanent noise-induced changes in distortion product otoacoustic emissions in $\mathrm{CBA} / \mathrm{CaJ}$ mice. Hear. Res. 156 , $31-43$.

Vetter, D.E., Mann, J.R., Wangemann, P., Liu, J., McLaughlin, K.J., Lesage, F., Marcus, D.C., Lazdunski, M., Heinemann, S.F., Barhanin, J., 1996. Inner ear defects induced by null mutation of the isk gene. Neuron 17 , $1251-1264$.

Woolf, N.K., Ryan, A.F., Bone, R.C., 1981. Neural phase-locking properties in the absence of cochlear outer hair cells. Hear. Res. 4, 335-346.

Zheng, W., Flavell, R.A., 1997. The transcription factor GATA-3 is necessary and sufficient for Th2 cytokine gene expression in CD4 T cells. Cell 89, 587-596. 